\title{
Individual response patterns in binary choice tasks with nondiscriminable stimuli '
}

\author{
MILTON H, HODGE AND WALTER H. RIEGE UNIVERSITY OF GEORGIA
}

The present experiment sought to determine whether individual $S$ s tend to employ repeatedly the same response patterns in binary choice tasks containing nondiscriminable stimuli and, if so, whether the response sequences when used to construct stimulus sequences improve the performance of other choice tasks. Information and frequency analyses of the response sequences of $48 \mathrm{~S}$ s showed moderate consistency of patterning within a light and within a tone task. Performance of a card task following the light and tone tasks was closely related to the task (light or tone) from which the stimulus sequence was obtained. The frequency analysis showed that repetition patterns were preferred by most $S_{s}$ but at the expense of alternation responses. The presentation of reinforcement in the card task did not improve performance over that observed in the light and tone tasks.

A number of studies (summarized by Tune, 1964) have shown that, under conditions of high stimulus similarity in binary choice tasks, human Ss exhibit systematic response preferences or patterns, e.g., repetition and alternation. When forced to respond to very similar stimuli, $S$ apparently falls back on the sequential dependencies inherent in his previous responses and/or generates a new pattern of responses. Since little is known about the conditions under which $\mathrm{S}$ ignores the stimuli of a task and generates stimulus-independent responses, it seems important to determine whether individual $S$ s utilize characteristic response patterns whenever they are presented with choice tasks in which the stimuli are nondiscriminable or very difficult to discriminate. If a $\mathrm{S}$ does employ particular response patterns in difficult choice tasks, a further question is whether performance of other choice tasks can be improved by constructing the stimulus sequences to follow exactly the response patterns previously generated by S. As compared with a random stimulus sequence, such a result would be expected since previous studies (Grant, Hake, \& Hornseth, 1951; Hake \& Hyman, 1953) have shown that Ss learn rather quickly the sequential dependencies of a stimulus series. The present experiment sought answers to these questions.

\section{METHOD}

Three binary choice tasks, each consisting of 300 trials, were performed individually by 48 male and female undergraduate students enrolled at the University of Georgia. The first two tasks utilized nondiscriminable tones and lights, respectively, with no feedback about wie correctness of the responses. In the tone task, $\mathrm{S}$ was asked to state whether the first or second tone had the higher pitch. In the light task, S was asked to indicate whether the right or left white light was the brighter. The Ss were not told that the two stimuli in each task were identical, but instead were led to believe that the stimuli were different. On each trial $S$ was given 4 sec. to make a decision. Half the Ss performed the two tasks in one of the two orders of administration and half of them in the other order.

Upon completion of the first two tasks, Ss were further divided into experimental and control groups, and then given the third task. In this task, S was asked to predict whether the next card would be marked with a blue or a yellow square. Following each trial, $S$ was told whether his prediction had been correct. Each $S$ in the experimental groups was given a stimulus sequence generated by him in either the first or second task. Thus, the third task was performed by four experimental groups, each containing eight Ss. The four groups were defined by the order of administration of the first two tasks and by the task from which the stimulus sequence of the third task was taken. Letting $\mathrm{L}$ and $\mathrm{T}$ represent the light and tone tasks, and $\mathrm{C}$ the card task, the four groups were $\mathrm{LTC}_{t}, \mathrm{LTC}_{1}$, $\mathrm{TLC}_{1}$, and $\mathrm{TLC}_{t}$. The $\mathrm{LTC}_{\mathrm{t}} \mathrm{Ss}$, for example, performed the light task first, the tone task second, and then the card task with the stimulus sequence taken from the response sequence generated in the tone task. The two control groups $\left(\mathrm{LTC}_{\boldsymbol{r}}\right.$ and $\mathrm{TLC}_{r}$ ), also containing eight Ss each, were given a random stimulus sequence in the card task.

\section{RESIJLTS}

The 900 responses of each $S$ were divided into three blocks of 100 responses in each task and examined for two kinds of consistency: (a) whether $S$ employed the same response pattern in eachblock of 100 within a task and (b) whether $S$ employed the same pattern in all three tasks. Overlapping groups of four responses (tetragrams) were selected as the unit of analysis because other investigators (e.g., Edwards, 1961) have shown that little additional information is obtained in longer patterns. Shorter patterns were not analyzed because all their information (in the technical sense) is contained in the tetragrams.

Information analysis. The tetragrams for each block of 100 responses were first subjected to a sequential information analysis (Attneave, 1959), producing nine $\mathrm{H}_{4}$ estimates ( 3 blocks by 3 tasks) for each $\mathrm{S}$. The maximum possible value of $\mathrm{H}_{4}$, corrected for lower-order dependencies, is $1.000 \mathrm{bit}$, indicating complete uncertainty in S's responses. A value less than 1.000 denotes the presence of patterning in the responses. The analysis showed that the individual $\mathrm{H}_{4}$ estimates ranged from 


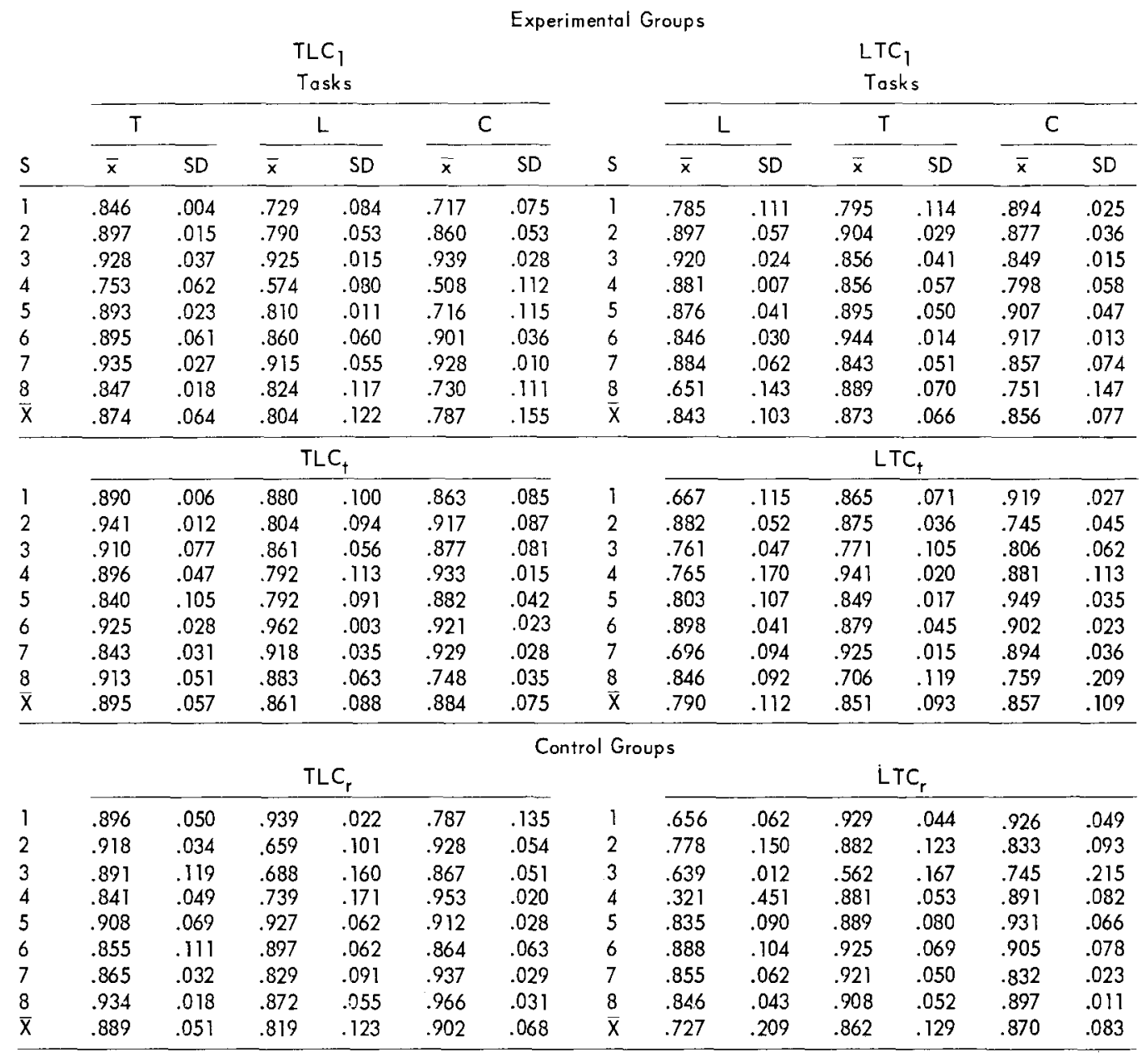

.135 to .988 . All Ss apparently introduced some degree of patterning in their responses, although some Ss showed more patterning than others. In an attempt to evaluate the consistency of the patterning, means and SDs of the trial blocks were computed for each $\mathrm{S}$ in each task. Table 1 shows that most of the individual means were fairly high, indicating moderate response patterning. The SD is a measure of the consistency of S's patterning, but it is not clear how large SD must be to reject the hypothesis of consistent patterning. One solution is to compare a S'S SD with arbitrary percentages of his mean. Of the 96 SDs (32 Ss by three tasks) computed for the experimental groups, $44 \%$ were smaller than a $5 \%$ deviation from the corresponding mean; $74 \%$ were less than a $10 \%$ deviation. For the two control groups, $25 \%$ and $69 \%$ of the SDs were less than $5 \%$ and $10 \%$ deviations from the means. Despite the arbitrariness of comparing an SD with a fixed percentage of a mean, the results suggest that most Ss are moderately consistent in their response patterning.

Table 1 also shows that in general the patterning is greatest in the light tasks. However, at the same time, the SDs indicate that the response patterns in the light tasks were not as stable as those in the tone tasks. Thus, although the response patterns were better defined in the light task, they were also more likely to change during a block of trials.

Performance of the card task in the experimental groups did not show a marked increase in patterning by using S's own response sequence as the stimulus sequence. In fact, a careful examination of Table 1 indicates that the degree of patterning in the card task is closely related to the task from which the stimulus sequence was obtained. On the other hand, relative to the card task in the control groups, performance of the experimental groups; particularly the $\mathrm{TLC}_{1}$ and TLC $_{t}$ groups, was somewhat enhanced by using S's response sequence as the stimulus sequence. It should be noted, however, that patterning in the card task of the control groups is very similar to that obtained in the tone tasks. This correlation may negate any meaningful comparison of the performance of the experimental and control groups on the card task. All the foregoing results are subject to still another 
limitation because of a fairly strong inverse relation $(r=-.69, p<.001)$ between the individual means and SDs (Table 1). The lower SDs associated with the tonetask means may be a function of the constraint imposed by the upper limit (1.000 bit) of the measurement scale. The SDs of means close to the limits of the scale must necessarily be smaller than the SDs of means nearer the midpoint.

Frequency analysis. Although information analysis has been widely used with sequential data, some investigators (e.g., Edwards, 1961; Tune, 1965) have argued that the procedure does not adequately discriminate among the various response patterns. To deal with this problem, Tune (1965) has suggested a frequency analysis of the 16 possible tetragram patterns in a binary sequence. On a chance basis, each of the 16 tetragrams would be expected to occur $6.25 \%$ of the time. Table 2 presents the mean percent occurrence of selected patterns summarized over Ss and trial blocks for the various experimental and control groups. The chance percentage for each type of pattern is 12.5 since each type is composed of two patterns. The first point to note is that repetition patterns generally occurred more often than any of the other patterns. Secondly, the departure from chance of the repetition patterns was more marked in the light than in the tone tasks, a result consistent with the finding in the information analysis that patterning was greatest in the light tasks. It should also be noted that the departures of the repetition patterns were usually accompanied by a decrease in the number of single alternation patterns. The occurrence of the triple and double alternation patterns was usually close to the chance percentage. Thirdly, to a greater degree than in the information analysis, the patterning in the card task for the experimental groups closely followed the response sequence of the task from which the stimulus sequence was taken. This effect can be seen in both the repetition and alternation patterns. Lastly, the column totals of Table 2 give the occurrence of the eight selected patterns relative to the 16 possible patterns. In all cases, the selected patterns account for more than $50 \%$ of the tetragrams, with the largest totals occurring in the light tasks.

Error analysis. As a final analysis, each S's errors on the card task were summarized for each block of 50 responses. An error represented a failure to predict correctly the color of the next card in the stimulus sequence. Table 3 shows the total errors of each $\mathrm{S}$ as a function of trial blocks and groups. An analysis of variance indicated significant differences among the task means $(F=165.9, \mathrm{df}=5 / 42, \mathrm{p}<.01)$. Further analysis of the task means with the NewmanKeuls test demonstrated that all the experimental groups except $\mathrm{TLC}_{\mathrm{t}}$ made significantly fewer errors than the control groups $(p<.01)$. The smallest number of errors were made by the $\mathrm{TLC}_{1}$ and $\mathrm{LTC}_{1} \mathrm{Ss}$, again the groups in which the light task is dominant.

\section{DISCUSSION}

Although the present results support Tune's (1964) hypothesis that Ss tend to produce nonrandom response sequences when forced to choose between nondiscriminable stimuli, the degree of patterning observed in the various tasks was not particularly strong. But, given the omission of informative feedback in the light and tone

Table 2. Mean Percent Occurrence of Selected Response Patterns

\begin{tabular}{|c|c|c|c|c|c|c|c|c|c|}
\hline \multirow[b]{4}{*}{ Pattern } & \multicolumn{6}{|c|}{ Experimental Group } & \multirow{3}{*}{\multicolumn{3}{|c|}{$\begin{array}{c}\text { Control Group } \\
\qquad \begin{array}{c}\text { LTC } \\
\text { Task }\end{array}\end{array}$}} \\
\hline & \multirow{2}{*}{\multicolumn{3}{|c|}{$\begin{array}{l}L_{T} \\
\text { Tosk }\end{array}$}} & \multirow{2}{*}{\multicolumn{3}{|c|}{$\begin{array}{l}\mathrm{LTC}_{f} \\
\text { Task }\end{array}$}} & & & \\
\hline & & & & & & & & & \\
\hline & L & $\mathrm{T}$ & $\mathrm{C}$ & $L$ & $\mathrm{~T}$ & C & $L$ & $T$ & $C$ \\
\hline $\begin{array}{l}\text { Repetition } \\
(0000,1111)\end{array}$ & 26.5 & 19.5 & 15.6 & 36.3 & 21.7 & 16.7 & 35.8 & 26.3 & 19.9 \\
\hline $\begin{array}{l}\text { Triple Alternation } \\
(1110,0001)\end{array}$ & 13.6 & 12.8 & 14.1 & 13.0 & 13.3 & 13.5 & 11.5 & 13.6 & 12.3 \\
\hline $\begin{array}{l}\text { Double Alternation } \\
(1100,0011)\end{array}$ & 10.8 & 13.1 & 19.5 & 10.8 & 11.5 & 12.5 & 9.7 & 12.4 & 15.0 \\
\hline $\begin{array}{l}\text { Single Altarnation } \\
(1010,0101)\end{array}$ & 7.5 & 9.8 & 6.1 & 4.2 & 10.0 & 9.1 & 3.7 & 7.4 & 7.8 \\
\hline \multirow[t]{2}{*}{ Total } & 58.4 & 55.2 & 55.3 & 64.3 & 56.5 & 51.8 & 60.7 & 59.7 & 58.0 \\
\hline & \multicolumn{3}{|c|}{$\mathrm{TLC}_{1}$} & \multicolumn{3}{|c|}{$\mathrm{TLC}_{t}$} & \multicolumn{3}{|c|}{$\mathrm{TLC}_{\mathrm{r}}$} \\
\hline $\begin{array}{l}\text { Repetition } \\
(0000,1111)\end{array}$ & 21.6 & 30.1 & 31.4 & 16.5 & 25.6 & 16.6 & 16.4 & 29.6 & 13.0 \\
\hline $\begin{array}{l}\text { Triple Alternation } \\
\text { (1110,0001) }\end{array}$ & 12.9 & 12.7 & 12.2 & 13.9 & 14.0 & 14.5 & 14.8 & 14.3 & 19.9 \\
\hline $\begin{array}{l}\text { Double Alternation } \\
(1100,0011)\end{array}$ & 12.3 & 12.4 & 13.2 & 14.2 & 12.9 & 16.4 & 14.8 & 13.0 & 18.0 \\
\hline $\begin{array}{l}\text { Single Alternation } \\
(1010,0101)\end{array}$ & 8.7 & 5.1 & 4.2 & 8.7 & 5.4 & 7.6 & 8.3 & 5.8 & 8.2 \\
\hline Total & 55.5 & 60.3 & 61.0 & 53.4 & 57.9 & 55.1 & 54.3 & 62.7 & 59.1 \\
\hline
\end{tabular}


Table 3. Number of Errors per Trial Block in the Card Task for the Experimental and Control Groups

Experimental Groups

TLC 1

Trial Blocks
$\mathrm{LTC}_{1}$

Trial Blocks

\begin{tabular}{|c|c|c|c|c|c|c|c|c|c|c|c|c|c|c|c|}
\hline$S$ & 1 & 2 & 3 & 4 & 5 & 6 & $\bar{x}$ & 5 & 1 & 2 & 3 & 4 & 5 & 6 & $\bar{x}$ \\
\hline 1 & 16 & 20 & 21 & 14 & 25 & 22 & 19.7 & 1 & 9 & 10 & 24 & 18 & 17 & 16 & 15.7 \\
\hline 2 & 27 & 17 & 25 & 25 & 21 & 29 & 24.0 & 2 & 24 & 18 & 23 & 20 & 31 & 22 & 23.0 \\
\hline 3 & 22 & 27 & 23 & 20 & 19 & 19 & 21.7 & 3 & 25 & 19 & 24 & 26 & 19 & 22 & 22.5 \\
\hline 4 & 14 & 18 & 9 & 14 & 9 & 5 & 11.5 & 4 & 25 & 23 & 29 & 24 & 27 & 28 & 26.0 \\
\hline 5 & 20 & 20 & 23 & 18 & 12 & 20 & 18.8 & 5 & 25 & 16 & 23 & 17 & 24 & 22 & 21.2 \\
\hline 6 & 26 & 25 & 30 & 20 & 22 & 24 & 24.5 & 6 & 22 & 25 & 20 & 27 & 21 & 26 & 23.5 \\
\hline 7 & 28 & 23 & 25 & 25 & 30 & 26 & 26.2 & 7 & 20 & 27 & 22 & 25 & 16 & 26 & 22.7 \\
\hline 8 & 18 & 12 & 14 & 15 & 19 & 22 & 16.7 & 8 & 37 & 14 & 16 & 21 & 24 & 18 & 21.7 \\
\hline $\bar{x}$ & 21 & 20 & 21 & 19 & 20 & 21 & 20.4 & $\bar{x}$ & 23 & 19 & 23 & 22 & 22 & 22 & 21.0 \\
\hline \multicolumn{8}{|c|}{$\mathrm{TLC}_{\dagger}$} & \multicolumn{8}{|c|}{$L T C_{t}$} \\
\hline 1 & 20 & 22 & 20 & 23 & 24 & 33 & 23.7 & 1 & 18 & 21 & 22 & 22 & 33 & 26 & 23.7 \\
\hline 2 & 19 & 17 & 26 & 26 & 25 & 20 & 22.2 & 2 & 20 & 21 & 18 & 21 & 24 & 25 & 21.5 \\
\hline 3 & 28 & 23 & 23 & 16 & 23 & 28 & 23.5 & 3 & 21 & 14 & 19 & 16 & 23 & 29 & 20.3 \\
\hline 4 & 23 & 24 & 28 & 23 & 23 & 17 & 23.0 & 4 & 26 & 24 & 24 & 21 & 21 & 21 & 22.9 \\
\hline 5 & 26 & 20 & 20 & 20 & 30 & 30 & 24.3 & 5 & 27 & 28 & 28 & 16 & 18 & 27 & 24.0 \\
\hline 6 & 30 & 26 & 23 & 30 & 21 & 18 & 24.7 & 6 & 21 & 18 & 27 & 22 & 17 & 26 & 21.8 \\
\hline 7 & 27 & 27 & 26 & 26 & 26 & 24 & 26.0 & 7 & 26 & 18 & 21 & 26 & 28 & 23 & 23.7 \\
\hline 8 & 25 & 20 & 25 & 24 & 25 & 26 & 24.2 & 8 & 32 & 26 & 19 & 17 & 17 & 13 & 20.7 \\
\hline $\bar{x}$ & 25 & 22 & 24 & 23 & 25 & 24 & 23.9 & $\bar{x}$ & 24 & 21 & 22 & 20 & 23 & 24 & 22.3 \\
\hline
\end{tabular}

Control Groups

\begin{tabular}{|c|c|c|c|c|c|c|c|c|c|c|c|c|c|c|c|}
\hline \multicolumn{8}{|c|}{$\mathrm{TLC}_{\mathrm{r}}$} & \multicolumn{8}{|c|}{$L T C_{r}$} \\
\hline 1 & 24 & 23 & 21 & 25 & 19 & 27 & 23.2 & 1 & 27 & 25 & 22 & 27 & 21 & 23 & 24.2 \\
\hline 2 & 29 & 31 & 26 & 23 & 26 & 29 & 27.3 & 2 & 18 & 26 & 22 & 18 & 22 & 19 & 20.8 \\
\hline 3 & 20 & 30 & 20 & 25 & 23 & 28 & 24.3 & 3 & 25 & 24 & 27 & 27 & 29 & 27 & 26 \\
\hline 4 & 21 & 24 & 22 & 25 & 22 & 28 & 23.7 & 4 & 30 & 25 & 23 & 24 & 29 & 25 & 26.0 \\
\hline 5 & 19 & 26 & 19 & 25 & 30 & 21 & 23.3 & 5 & 20 & 29 & 33 & 24 & 22 & 32 & 26.7 \\
\hline 6 & 20 & 23 & 25 & 24 & 23 & 21 & 22.7 & 6 & 31 & 35 & 29 & 17 & 29 & 27 & 28.0 \\
\hline 7 & 22 & 30 & 24 & 27 & 28 & 27 & 26.3 & 7 & 21 & 27 & 30 & 25 & 28 & 19 & 25.0 \\
\hline 8 & 24 & 22 & 26 & 27 & 24 & 27 & 25.0 & 8 & 20 & 28 & 32 & 27 & 23 & 28 & 26.3 \\
\hline $\bar{x}$ & 22 & 26 & 23 & 25 & 24 & 26 & 24.5 & $\frac{x}{x}$ & 24 & 27 & 27 & 24 & 25 & 25 & 25.4 \\
\hline
\end{tabular}

tasks, perhaps stronger patterning should nothave been expected. The preceding conclusion is a reasonable interpretation of the information analysis. However, the frequency analysis provides a more optimistic view of the results. Repetition was clearly the preferred mode of responding; in some of the light tasks repetition patterns occurred three times more frequently than expected by chance, and interestingly, at the expense of single alternation responses. Further research, of course, would be necessary to establish whether there is a systematic trading relation between repetition and alternation responses in binary choice tasks. A careful experimental study of the proposed relation and the conditions which influence it might show that repetition and alternation are the major ways in which the naive $\mathrm{S}$ attempts to cope with choice tasks possessing high stimulus uncertainty. The fact that some previous studies (e.g., Dale, 1965) of response preferences have found strong repetition tendencies while others (e.g., Jarvik, 1951) have observed alternation responses may represent nothing more than the presence of different levels of the same variables.

A major goal of the present experiment was to deter- mine whether individual Ss tend to employ repeatedly a particular pattern of responses. The information analysis suggests that the answer depends upon the nature of the experimental task and upon the criterion adopted for evaluating response consistency. With a strict criterion, $44 \%$ of the individual SDs in the experimental groups (Table 1) were smaller than a $5 \%$ deviation from the corresponding means. With a less stringent standard, $74 \%$ of the SDs were less than a $10 \%$ deviation from the means. Somewhat smaller percentages were obtained in the control groups, primarily as the result of larger SDs in all tasks. ${ }^{2}$ However, both the information and frequency analyses also showed that the strength and consistency of the patterning were a function of the particular task. The patterning was stronger but less consistent in the light tasks than in the tone tasks. Excluding sense modality, the most obvious difference between the two tasks is the spatial (light) vs. temporal (tone) presentation of the two stimuli. Why the mode of stimulus presentation should produce systematic differences in the strength and consistency of the patterning is not clear. But, its importance is also supported by the finding that 
the patterning in the card task of the experimental groups tended to parallel the patterning of the task from which the card-task stimulus sequence was obtained.

It was somewhat surprising that the combination of informative feedback and stimulus sequences modelled after earlier response sequences did not produce a marked improvement in the card task of the experimental groups. Both variables should have encouraged strong patterning. The close agreement between cardtask performance and that of the task from which the stimulus sequence was taken suggests that reinforcement in the card task was simply not effective. That the performance of a card task cannot be influenced by reinforcement is contradicted by the numerous probability learning experiments (Estes, 1959) which have employed such a task. A more likely, though admittedly vague, hypothesis is that performance of the light and tone tasks established strong, and perhaps rigid, response strategies which Ss were unable or unwilling to abandon in the card task. Another possibility is that the effects of reinforcement are sufficiently delayed so that by the time $S$ is able to modify his responses the stimulus sequence has changed. Consequently, summation of the effects of the sequential dependencies and reinforcement does not occur because the responses implied by the reinforcement variable are no longer compatible with those suggested by the sequential dependencies.

Relative to the control groups, all the analyses indicated that performance of the card task by the experimental groups was superior. However, an additional control group given stimulus sequences following previous response sequences but without informative feedback would have made the comparisons between the experimental and control groups more meaningful. Such a group would have permitted an evaluation of the effects of reinforcement on the card task.

Finally, the similarity of the results obtained in the frequency and information analyses suggests that both are useful techniques for analyzing sequential patterns. The information analysis provides a single, overall index of the patterning, which is not supplied by the frequency analysis, but, at the same time, the frequency technique yields information about specific patterns not differentiated among by the information analysis.

\section{References}

Attneave, F. Applications of information theory to psychology. New York: Holt, 1959.

Dale, H. C. A. Recency in two-choice guessing tasks. Brit. J. Psychol., 1965, 56, 33-44

Edwards, W. Probability learning in 1000 trials. J. exp. Psychol. $1961,62,385-390$.

Estes, $W . K$. The statistical approach in Iearning theory. In $S$. Koch (Ed.), Psychology: A study of a science. Vol. 2. General systematic formulations, learning, and special processes. New York: MeGraw-Hill, 1959. Pp. 380-492.

Grant, D. A., Hake, H. W., \& Homseth, J. P. Acquisition and extinction of a verbal conditioned response with differing percentages of reinforcement. 7 . exp. Psychol., 1951, 41, 1-5.

Hake, H. W., \& Hyman, R. Perception of the statistical structure of a random series of binary symbols. J. exp. Psychol., 1953, $45,64-74$.

Jarvik, M. E. Probability learning and a negative recency effect in the serial anticipation of alternative symbols. J. exp. Psychol., 1951, 41, 291-297.

Tune, G. S. Response preferences: A review of some relevant literature. Psychol. Bull., 1964, 61, 286-302.

Tume, $\boldsymbol{G} . \mathbf{S}$. The equiprobable hypothesis as an initial response tendency, J. gen. Psychol, 1965, 72, 263-267.

\section{Notes}

1. The present report is based on a thesis by the second author submitted in partial fulfillment of the requirements for the M.S. degree at the University of Georgia. We are grateful to Richard c. Hazen for his helpful comments.

2. No explanation can be offered for the larger SDs of the control groups, but an inspection of Table 1 suggests that the control Ss may not belong to the same population as the experimental Ss.

(Accepted for publication October 8. 1966.) 